\title{
¿Está mediada la gravedad delictiva y cronicidad de los delincuentes juveniles por la competencia cognitivo-comportamental?**
}

\author{
Is Offence Severity and the Chronicity of Juvenile Delinquency \\ Mediated by Cognitive-Behavioural Competence?
}

Recibido: marzo 28 de 2013 | Revisado: junio 9 de 2013 | Aceptado: abril 14 de 2014

\author{
FrANCISCA FARIÑA ** \\ MARÍA José VÁzQUEZ \\ Universidad de Vigo, Galicia, España \\ RAMÓN ARCE*** \\ Universidad de Santiago de Compostela, Galicia, España
}

doi:10.11144/Javeriana.UPSY13-3.emgd

Para citar este artículo: Fariña. F., Vásquez, M. J. \& Arce, R. (2014). iEstá mediada la gravedad delictiva y cronicidad de los delincuentes juveniles por la competencia cognitivo-comportamental? Universitas Psychologica, 13(3), 881-894. http:// dx.doi.org/10.11144/Javeriana.UPSY13-3.emgd

* Esta investigación fue financiada por el Ministerio de Asuntos Exteriores y Cooperación de España. Secretaría de Estado de Cooperación Internacional (Código: A/026189/09).

** Departamento de Análise e Intervención PsicoSocioEducativa (A.I.P.S.E.).

**** Departamento de Psicología Social, Básica y Metodología, Universidad de Santiago de Compostela, Galicia, España. Facultad de Psicología. Campus Vida, s/n. E-15782 Santiago de Compostela. Correo electrónico: ramon.arce@usc.es

\section{RES U MEN}

El estudio de la relación de los problemas de conducta con la competencia cognitiva y comportamental se ha convertido en un referente continuo en la literatura científica. A este respecto, los modelos de Riesgo-NecesidadesResponsividad (RNR) y de la criminología del desarrollo prevén que el nivel de problemas de conducta esté asociado al grado de desarrollo de la competencia cognitiva y comportamental. Desde una perspectiva legal, los problemas de conducta se identifican con la delincuencia, estableciéndose niveles según la gravedad delictiva (delitos con vs. sin violencia) y del delincuente (primarios vs. reincidentes). Para comprobar si estos niveles se reflejan en la competencia cognitiva y comportamental, 283 adolescentes que estaban cumpliendo una condena judicial, 139 (68 reincidentes y 71 primarios) habían cometido un delito violento y 144 sin violencia ( 77 reincidentes y 67 primarios), fueron evaluados en las medidas de referencia de la competencia cognitiva (e. g., regulación emocional, autoconcepto, atribución de la responsabilidad, resolución de problemas) y comportamental. Los resultados mostraron que la gravedad delictiva es independiente de la competencia cognitiva y comportamental y que la delincuencia crónica se relaciona con menor competencia. De estos resultados se derivan consecuencias para el tratamiento de los delincuentes juveniles y para la duración de las condenas que se discuten.

Palabras clave

problemas de conducta; delincuencia juvenil; comportamiento antisocial; violencia; reincidencia; competencia cognitiva

\section{A B S T R A C T}

The correspondence between juvenile delinquency and cognitive-behavioural competence has been the subject of ongoing concern in the scientific literature. Developmental criminology and Risk-Need-Responsivity (RNR) models have associated offence severity and the chronicity of juvenile delinquency to the degree of cognitive-behavioural development. In terms of the juvenile justice system, delinquent behaviour is classified according to offence severity (violent vs. non-violent offences), and the type of offender (first-time offender vs. chronic offender). In order to assess the nexus between chronic delinquent behaviour and the degree of cognitivebehavioural competence, 283 juveniles serving custodial sentences, 139 for violent offences (71 first-time offenders and 68 reoffenders), and 144 for non-violent offences ( 77 reoffenders and 67 first-time offenders), were 
assessed in terms of cognitive (e.g., regulation emotional, self-concept, attribution of responsibility, problem-solving skills) and behavioural competence. The results revealed that offence severity was not mediated by the level of cognitive-behavioural competence, and chronic delinquency was linked to low levels of competence. The results are discussed in the light of juvenile delinquency treatment programmes and sentencing.

Keywords

conduct problems; juvenile offenders; antisocial behavior; violence; recidivism; cognitive competence

\section{Introducción}

La literatura científica ha venido informando de una alta prevalencia de problemas de conducta en adolescentes, al tiempo que equiparando, bajo esa etiqueta, un amplio abanico de comportamientos impulsivos, que van desde una intranscendente rabieta, hablar negativamente de otro hasta la agresión a personas (Gázquez, Pérez-Fuentes, Carrión \& Santuiste, 2010; McMahon, Wells \& Kotler, 2006). Este hecho ha conllevado a que en la investigación se tomaran como equivalentes y generalizables diseños y resultados de unos comportamientos a otros. Sin embargo, las implicaciones legales, sociales, para el tratamiento, de riesgo de carrera delictiva o peligrosidad de comportamientos negativistas/ desafiantes (p. ej., rabietas, pataletas, gritos); disruptivos (aquellos que implican la interrupción o desajuste en el desarrollo evolutivo); antisociales (aquellos que violan las normas sociales, pero no las legales); delictivo no violentos (aquellos que violan el código penal sin enfrentamiento con la víctima); o delictivo-violentos (aquellos que contravienen el código penal y que implican el uso de la violencia) son claramente diferenciadas.

Del mismo modo, desde una perspectiva de salud mental, en el modelo clínico-biológico (American Psychiatric Association [APA], 2013; Howard, 2009; Rhee \& Waldman, 2009), se incluyen dichos comportamientos bajo un trastorno de personalidad, el trastorno disocial. En este trastorno y bajo el paraguas de comportamientos antisociales, se entremezclan comportamientos propiamente antisociales, tales como las fugas de casa de noche (Criterio A14); delictivo-violentos, tal como la agresión a personas o animales (Criterios A1 a A7); delictivos no violentos, como el robo sin enfrentamiento con la víctima (Criterio A12); o disruptivos en el aula (como hacer novillos en la escuela, Criterio A15). En relación con los tipos, la APA (2013) en el DSM-V diferencia, en función de la edad de inicio del trastorno, entre tipo de inicio infantil y tipo de inicio adolescente (frente al infantil, se caracteriza por ser menos persistente, con menos comportamientos agresivos y con menos probabilidad de desarrollo en la edad adulta de un trastorno antisocial de la personalidad).

Adicionalmente, y como trastorno de personalidad requiere de continuidad y estabilidad, esto es, que sea un patrón de comportamiento repetitivo y persistente de comportamiento. Aunque no existe consenso sobre la conveniencia de emplear el término psicópata para referirse a adolescentes, motivado fundamentalmente por razones éticas, metodológicas y de desarrollo (Marsee, Silverthorn \& Frick, 2005), la relación entre rasgos psicopáticos y problemas de conducta es robusta (Andershed, Gustafson, Kerr \& Stattin, 2002; Declercq, Markey, Vandist \& Verhaeghe, 2009; Frick, Stickle, Dandreaux, Farrel \& Kimonis, 2005; Lynam, Miller, Vachon, Loeber \& Stouthamer-Loeber, 2009; Marsee et al., 2005; McMahon, Witkiewitz \& Kotler, 2010). A diferencia del trastorno disocial, la psicopatía no pivota únicamente en torno al comportamiento, sino a dimensiones, entre las que sobresalen el narcisismo, insensibilidad emocional e impulsividad, que se toman como predictores de los problemas de conducta (Frick et al., 2005).

Sin embargo, desde un punto de vista de la justicia penal, solo los actos delictivos son objeto de intervención, entendiendo por estos aquellos cuya acción constituya un delito con arreglo a la legislación nacional o al derecho internacional. Por consiguiente, los comportamientos antisociales, los negativistas/desafiantes y los disruptivos, que no entrañen infracción alguna recogida en el código penal, esto es, que no constituyan un delito, no son objeto de acción judicial. Asimismo, la psicopatía no es equiparable a la delincuencia legal, ya que no todos los delincuentes son psicópatas, ni todos los psicópatas son delincuentes (Arce, Fariña \& 
Vázquez, 2011; Halty, Martínez, Requena, Santos \& Ortíz, 2011; Muñoz, Kerr \& Besic, 2008; Serin \& Amos, 1995). Los actos delictivos son clasificados penalmente (p. ej., Ley Orgánica 5/2000; Ley Orgánica 8/2006) en dos grandes categorías: a) gravedad delictiva, que los divide en delitos cometidos con el uso de violencia y sin el uso de violencia y b) cronicidad, discriminando entre delincuentes primarios y reincidentes o crónicos. Esta clasificación legal entra en contradicción con las científicas o de salud mental que requieren del cumplimiento de unos criterios y de un patrón de comportamiento repetitivo y persistente, desestimándose así el acto ocasional (delincuente primario), cuando puede que sean escalones distintos de un recorrido de la normalidad a la delincuencia o en la gravedad de esta.

Por su parte, en el DSM-V se recogen tres niveles de gravedad, en función del número de criterios cumplidos y del daño causado a otros: leve (pocos o ningún criterio excede los requeridos para el diagnóstico y los problemas de conducta no son delictivos); moderado (intermedio entre leve y moderado y actos delictivos sin violencia/daños a las personas) y grave (muchos problemas de conducta, incluyendo violencia/daños a las personas). En suma, ciencia y salud mental por una parte, y ley, por otra, usan clasificaciones no intercambiables.

Los modelos de cascada/escalada en el desarrollo (Hawley, 2003; Masten \& Cicchetti, 2010) e historia natural del delito (Moffitt, 1993) prevén que la persistencia y el nivel de problemas de conducta (en escalada y persistencia desde conductas prosociales a delictivo-violentas) está asociado con el nivel de desarrollo de la competencia cognitiva y comportamental. Similarmente, los modelos RiskNeed-Responsivity (RNR) e Integrated Differential Intervention Model (IDIM) relacionan los problemas de conducta y (re)incidencia delictiva con la acumulación de factores de riesgo, dirigiendo la intervención a la subsanación de los déficits en habilidades, cogniciones y emociones y a un ajuste a las necesidades del sujeto (Andrews \& Bonta, 2010; Fréchette \& LeBlanc, 1987).

En suma, desde una óptica del desarrollo se liga (in) competencia y desviación. La investigación de campo apoya esta conexión. Así, Contreras, Moli- na y Cano (2011) encontraron que los delincuentes juveniles reincidentes compartían una menor competencia cognitiva y comportamental que los primarios; Arce et al. (2011) que los adolescentes en riesgo de desviación social que los normativos; Rodríguez et al. (2011) que los delincuentes reincidentes se inician más temprano en los comportamientos delictivos que los no reincidentes y Arce, Seijo, Fariña y Mohamed-Mohand (2010) que los delincuentes juveniles reincidentes eran más incompetentes que los primarios, estos que los antisociales, y estos últimos que los normativos.

Esta relación entre nivel de problemas de conducta, por una parte y niveles de desarrollo de la competencia cognitiva y comportamental, por otra, también puede tener su correspondencia con la gravedad del delito y reincidencia. La variabilidad en las tasas de reincidencia es una constante en la literatura, así como que ésta se relaciona de un modo robusto con los moderadores tipo de delito, cronicidad y de tratamiento (McGuire, 2008; Redondo, Sánchez-Meca \& Garrido, 2002). Sucintamente, a mayor gravedad del delito, mayor tasa de reincidencia; los delincuentes habituales, frente a los primarios, tienen una mayor probabilidad de reincidencia y los tratamientos basados en el entrenamientos de destrezas conductuales y cognitivoconductuales, son los más efectivos en la prevención de la reincidencia.

A modo de ejemplo, recientemente el Indiana Department of Correction (2010) desveló una tasa de reincidencia general entre delincuentes juveniles del $39.2 \%$, oscilando, en función de la gravedad del delito, desde los menos graves, como la falta cuya tasa se cifró en el 22\%, hasta los más graves, como el uso de armas, que alcanzó el 47.8\%; Redondo et al. (2002) hallaron en un metaanálisis que la probabilidad de reincidencia delictiva entre los delincuentes no sometidos a tratamiento era del $60.5 \%$, reduciéndose entre aquéllos objeto de tratamiento un $21 \%$, especialmente con programas conductuales y cognitivo-conductuales; en tanto los estudios de cohortes y de desarrollo de la carrera delictiva precisan que un número pequeño de delincuentes juveniles desarrollan una carrera delictiva (crónicos frente a los ocasionales o primarios), siendo 
los autores de la mayoría de los actos delictivos: los delincuentes crónicos, entre el 5 y el 10\% del total, son responsables, según la categoría delictiva, aproximadamente desde el 60 a más del 80\% de total de delitos (Elrod \& Ryder, 2011).

Según se adopte un modelo clínico-biológico o de competencia, las variables de medida son unas u otras. Así, la asunción de un modelo clínico-biológico requiere de una evaluación de los trastornos clínicos y síntomas asociados y de la manipulación de las respuestas (simulación y mentira). Por su lado, los modelos de competencia cognitiva y comportamental implican la potenciación de los factores protectores de la desviación social y la neutralización de los factores de riesgo. Estos varían de unos contextos y submodelos a otros, siendo los fundamentales las distorsiones cognitivas (p. ej., no asunción de la responsabilidad en los actos delictivos; negación de los hechos) (Maruna \& Mann, 2006); los mecanismos internos subyacentes a la delincuencia (p. ej., ira/hostilidad) (Binswanger et al., 2010; Novo, Fariña, Seijo \& Arce, 2012); las destrezas cognitivas (p. ej., autocontrol), comportamentales (p. ej., técnicas de resolución de problemas) y sociocomunitarias (p. ej., adaptación escolar, familiar) (Lösel \& Farrington, 2012).

En suma, la hipótesis que relaciona niveles de incompetencia cognitiva y comportamental con niveles de problemas de conducta, parece robusta, al tiempo que puede estar mediada por otros moderadores, tales como la gravedad del delito y la cronicidad o carrera delictiva. Como consecuencia de este estado de la literatura, nos hemos planteado un estudio de cam- po en el que someter a prueba si la clasificación legal de la gravedad delictiva (con vs. sin violencia) y del delincuente (primarios vs. reincidentes), así como su interacción, tiene un reflejo en la competencia cognitiva y comportamental, tomando como medidas de ésta aquellas que se mostraron sensibles en la discriminación entre poblaciones de adolescentes delincuentes, con comportamientos antisociales y normativos (Arce et al., 2010; Arce et al., 2011; Contreras et al., 2011; Rodríguez et al., 2011).

Adicionalmente, también se contrastará este mismo diseño en el modelo clínico-biológico (APA, 2013): la simulación, mentira, y los trastornos y síntomas asociados.

\section{Método}

\section{Participantes}

En este estudio participaron 283 adolescentes, con una edad media de 17.17 años $(E E M=0.08)$, que estaban cumpliendo una condena judicial (véase en la Tabla 1 la distribución de medidas) con una duración media de 470.77 días $(E E M=42.12)$, de los cuales 145 eran reincidentes y 138 primarios. Según el tipo de delito, 139 (68 reincidentes y 71 primarios) habían cometido un delito violento y 144 sin violencia (77 reincidentes y 67 primarios).

\section{Procedimiento y diseño}

Las evaluaciones se llevaron a cabo en pases colectivos en los respectivos centros u organismos en los

TABLA 1

Distribución de la población por medida judicial en cumplimiento

\begin{tabular}{lcc}
\hline \multicolumn{1}{c}{ Delito } & Frecuencia & Porcentaje \\
\hline Tareas socioeducativas & 7 & 2.5 \\
Prestaciones en beneficio de la comunidad & 4 & 1.4 \\
Libertad vigilada & 31 & 11 \\
Tratamiento ambulatorio & 2 & 0.7 \\
Asistencia a un centro de día & 15 & 5.3 \\
Internamiento en centro semiabierto & 31 & 11 \\
Internamiento en centro cerrado & 167 & 59 \\
Internamiento terapéutico & 26 & 9.2 \\
\hline
\end{tabular}

Fuente: elaboración propia 
que se les estaba administrando la medida judicial, contando para ello con el permiso de las autoridades administrativas, de una comisión ética del centro $\mathrm{u}$ organismo creada para el efecto y del propio infractor. Los datos fueron tratados garantizando el anonimato de los participantes, las normativas de protección de datos y de los derechos de los jóvenes, y se obtuvieron en sesiones individuales de evaluación. Dichas sesiones no superaron en ningún caso, a fin de controlar los efectos del cansancio, los 40 minutos, haciendo uso de las sesiones que fueran necesarias en cada caso concreto. El orden de obtención de las medidas fue contrabalanceado siguiendo para ello una rotación estándar.

La metodología de investigación empleada fue del tipo cuasiexperimental y en un ambiente natural. Se planificó un diseño factorial completo 2 (tipo de delito: con vs. sin violencia), X 2 (reincidencia: primario vs. reincidente) sobre un conjunto de variables que se relacionan con la (in)competencia cognitiva y comportamental. Los niveles del factor tipo de delito, con vs. sin violencia, vienen definidos por las acciones y tipología delictivas. Así, los delitos con violencia se definen como aquellos cuya figura delictiva implica un asesinato, intento de homicidio, homicidio, homicidio imprudente, lesiones, violencia sobre familiares, sobre la pareja, lesiones, abusos sexuales, agresión sexual, robo con intimidación, robo con violencia, amenazas o bullying. Por su lado, son delitos sin violencia son aquellos en los que no hay violencia, intimidación o grave riesgo para la vida o integridad física de las personas, in- cluyéndose aquí las restantes categorías delictivas. El segundo factor, la reincidencia, fue informado por los técnicos que les administraban el tratamiento judicial sobre la base de las decisiones judiciales y los expedientes de los participantes.

Sometida a análisis la sensibilidad del diseño hallamos que, con una muestra de 283 delincuentes juveniles sentenciados por actos delictivos, la probabilidad de detección (1- $\beta$ ) de diferencias de medias significativas $(\alpha<0.05)$ para un tamaño del efecto medio $(f=0.25)$ mediante un MANOVA para 4 grupos comparados entre 3 y 18 variables, oscila entre el 99 y el 100\%. En suma, la sensibilidad del diseño es prácticamente total.

\section{Instrumentos y variables de medida}

Como instrumentos de medida, cuyas estimaciones de la fiabilidad con los participantes en el estudio pueden verse en la Tabla 2 , se tomaron para la evaluación del autoconcepto el Cuestionario de Autoconcepto (AFA-A) (Musitu, García \& Gutiérrez, 1997); de la inteligencia emocional, la adaptación española de la Trait Meta-Mood Scale (TMMS) (Fernández-Berrocal, Extremera \& Ramos, 2004); del Locus de Control, la escala de Rotter (1966); de la socialización, la Batería de Socialización (BAS3) (Silva \& Martorell, 1989); de la adaptación, el Test Autoevaluativo Multifactorial de Adaptación Infantil (TAMAI) (Hernández, 2002); y de las estrategias de afrontamiento, la Escala de Afrontamiento para Adolescentes (ACS) (Frydenberg

TABLA 2

Fiabilidad de los instrumentos de medida con la muestra de este estudio

\begin{tabular}{|c|c|}
\hline Instrumento & alpha de Cronbach \\
\hline Autoconcepto (AFA) & 0.87 \\
\hline Trait Meta-Mood Scale (TMMS) & 0.82 \\
\hline SCL-90-R & 0.91 \\
\hline Escala de Locus de Control de Rotter & 0.71 \\
\hline Batería de Socialización-BAS/3 & 0.82 \\
\hline Test Autoevaluativo Multifactorial de Adaptación Infantil (TAMAI) & 0.79 \\
\hline Escala de Afrontamiento para Adolescentes (ACS) & 0.96 \\
\hline
\end{tabular}

Nota. Datos referidos al total de la escala

Fuente: elaboración propia 
\& Lewis, 2000). Como medio de evaluación de la simulación se siguió el protocolo validado para este efecto por Vilariño, Fariña y Arce (2009) de modo que si el GSI $\geq 1.47$, el PSDI $\geq 2.71$ o el PST $\geq 54$ del SCL-90-R, la probabilidad de que se esté ante un caso de simulación es prácticamente total en poblaciones no clínicas.

\section{Análisis de datos}

Para el contraste de medias se procedió con los ANOVA o los MANOVA, según procediera. En la F multivariada se tomó como estadístico multivariante la traza de Pillia-Bartlett, porque se ajusta mejor a las Ciencias del Comportamiento y es más robusta frente a la heterogeneidad de varianza y la violación de la normalidad multivariable $(\mathrm{Ol}$ son, 1976). Para la interpretación de la potencia se siguieron los criterios de Cohen (1988): tamaño pequeño $\left(\eta^{2}<0.058\right)$ moderado $\left(\geq 0.059 \eta^{2}\right.$ $<0.137)$ y grande $\left(\eta^{2} \geq 0.137\right)$. El contraste de probabilidades se llevó a cabo por medio del estadístico ji cuadrado y la potencia por medio de phi. En la interpretación de la potencia se tomaron los criterios de Cohen (1988): tamaño pequeño $(\Phi<$ $0.3)$, moderado $(\geq 0.3 \Phi<0.5)$ y grande $(\Phi \geq 0.5)$. Los tamaños de los efectos han de ser considerados a la luz del contexto de evaluación y de las predicciones de los modelos. Sucintamente, la literatura no prevé, en general, diferencias en la competencia cognitiva y comportamental entre las diversas tipologías de delincuentes juveniles. Por ello, tamaños del efecto moderados e incluso pequeños serían de gran valor teórico y, sobre todo, para la implementación de programas de prevención y tratamiento.

\section{Resultados}

\section{Inteligencia emocional}

Realizado un MANOVA 2 (tipo de delito: con violencia vs. sin violencia) X 2 (reincidencia: primarios vs. reincidentes), los resultados mostraron efectos no significativos en la inteligencia emocional para el factor tipo de delito, $F(3,277)=0.06, n s, \eta_{p}{ }^{2}=$ $0.001,1-\beta=0.061$, para el factor reincidencia, $F(3$,
277) $=0.75, n s, \eta_{\mathrm{p}}{ }^{2}=0.008,1-\beta=0.209$, y para la interacción de ambos, $F(3,277)=0.48, n s, \eta_{p}^{2}=$ $0.005,1-\beta=0.146$.

\section{Inadaptación}

Efectuado un MANOVA 2 (tipo de delito: con violencia vs. sin violencia) X 2 (reincidencia: primarios vs. reincidentes), arrojó un efecto no significativo para el factor tipo de delito, $F(4,276)=$ $0.01, n s, \eta_{\mathrm{p}}{ }^{2}=0.005,1-\beta=0.13$, significativo para el factor reincidencia, $F(4,276)=3.19, p<0.05$, $\eta_{\mathrm{p}}{ }^{2}=0.044,1-\beta=0.822$, y no significativo para la interacción de ambos, $F(4,276)=0.68, n s, \eta_{p}^{2}$ $=0.01,1-\beta=0.219$. Así, el factor reincidencia, que explica el $4.4 \%$ de la varianza, un tamaño del efecto pequeño, media diferencias significativas en la inadaptación de los delincuentes que se concretan en inadaptación escolar, $F(1,279)=4.28, p<$ $0.012, \eta_{\mathrm{p}}{ }^{2}=0.017,1-\beta=0.54$, y social, $F(1,279)=$ $7.29, p<0.001, \eta_{p}^{2}=0.028,1-\beta=0.767$, de modo que los reincidentes están más inadaptados escolar (Medias $=14.99$ y 12.64) y socialmente (Medias $=$ 12.54 y 10.34) que los primarios. En consecuencia, los delincuentes juveniles reincidentes manifiestan más conductas disruptivas en el aula, menos esfuerzo y aplicación en el aula y una mayor actitud desfavorable hacia el aprendizaje escolar, así como una mayor falta de control y restricción en las relaciones sociales.

\section{Socialización}

Efectuado un MANOVA 2 (tipo de delito: con violencia vs. sin violencia) X 2 (reincidencia: primarios vs. reincidentes), los resultados mostraron un efecto significativo en la socialización sí para el factor reincidencia, $F(5,275)=3.39, p<0.01$, $\eta_{p}{ }^{2}=0.058,1-\beta=0.903$, no significativo para el factor tipo de delito, $F(5,275)=0.63, n s, \eta_{p}{ }^{2}=$ $0.011,1-\beta=0.227$, y para la interacción de ambos, $F(5,275)=0.6, n s, \eta_{p}{ }^{2}=0.011,1-\beta=0.218$. Ciertamente, el factor reincidencia, que da cuenta del $6.8 \%$ de la varianza, un tamaño del efecto moderado, media diferencias en la socialización de los delincuentes juveniles, específicamente en 
el autocontrol social, $F(1,279)=6.97, p<0.01$, $\eta_{\mathrm{p}}{ }^{2}=0.024,1-\beta=0.749$. Concretamente, los reincidentes acatan en menor medida las normas y reglas sociales que los primarios (Medias $=8.36 \mathrm{y}$ 9.50 , respectivamente) sustituyendo el respeto por las normas por conductas agresivas, impositivas, de terquedad e indisciplina.

\section{Autoconcepto}

Practicado un MANOVA 2 (tipo de delito: con violencia vs. sin violencia) X 2 (reincidencia: primarios vs. reincidentes), los resultados mostraron un efecto significativo en el autoconcepto para el factor reincidencia, $F(4,276)=5.58, p<0.001, \eta_{p}{ }^{2}$ $=0.075,1-\beta=0.977$, pero no así para el factor tipo de delito, $F(4,276)=1.16, n s, \eta_{p}^{2}=0.017,1-\beta=$ 0.364 , ni para la interacción de ambos, $F(4,276)=$ $0.54, n s, \eta_{p}{ }^{2}=0.008,1-\beta=0.18$. En otras palabras, la reincidencia delictiva, que da cuenta del $7.5 \%$ de la varianza, un tamaño del efecto moderado, media el autoconcepto de los delincuentes juveniles; concretamente, del autoconcepto académico, $F(1$, 279) $=8.82, p<0.003, \eta_{\mathrm{p}}{ }^{2}=0.016,1-\beta=0.841, \mathrm{y}$ familiar, $F(1,279)=13.25, p<0.001, \eta_{p}^{2}=0.103$, $1-B=0.952$. Resumidamente, los infractores primarios participan de una identidad escolar (Medias $=20.32$ y 21.67) y familiar (Medias $=9.81$ y 11.08) más baja que los reincidentes.

\section{Afrontamiento}

Llevado a cabo un MANOVA 2 (tipo de delito: con violencia vs. sin violencia) X 2 (reincidencia: primarios vs. reincidentes), los resultados mostraron, en las estrategias de afrontamiento, un efecto no significativo para el factor tipo de delito, $F(18$, $262)=1.1, n s, \eta_{p}^{2}=0.07,1-\beta=0.759$, para el factor reincidencia, $F(18,262)=1.47, n s, \eta_{p}^{2}=0.091$, $1-\beta=0.897$, y para la interacción de ambos, $F(18$, $262)=1.61, n s, \eta_{\mathrm{p}}{ }^{2}=0.1,1-\beta=0.929$. En suma, primarios y reincidentes comparten las capacidades para la resolución de problemas, al tiempo que la comisión delitos con o sin violencia tampoco se relaciona con una competencia diferencial en la resolución de problemas.

\section{Procesos de atribución}

Un ANOVA 2 (tipo de delito: con violencia vs. sin violencia) X 2 (reincidencia: primarios vs. reincidentes), no mostró diferencias significativas en los procesos atribucionales mediadas por el factor tipo de delito, $F(1,279)=0.09$, ns, $\eta_{\mathrm{p}}{ }^{2}=0,1-\beta=0.061$, por el factor reincidencia, $F(1,279)=0.75, n s, \eta_{p}^{2}=$ $0.003,1-\beta=0.139$, ni por la interacción de ambos, $F(1,279)=1.01, n s, \eta_{p}{ }^{2}=0.004,1-\beta=0.17$, esto es, son factores independientes. Así pues, ni la reincidencia ni el tipo de delito implican una asunción diferente de la responsabilidad de los actos.

\section{Distrés psicológico}

Los resultados de un MANOVA 2 (tipo de delito: con violencia vs. sin violencia) X 2 (reincidencia: primarios vs. reincidentes), no arrojaron un efecto multivariado significativo en el distrés psicológico para el factor reincidencia, $F(3,277)=2.61, n s, \eta_{p}{ }^{2}$ $=0.027,1-\beta=0.636$, para el factor tipo de delito, $F(3,277)=0.29, n s, \eta_{\mathrm{p}}{ }^{2}=0.003,1-\beta=0.105, \mathrm{y}$ para la interacción de ambos factores, $F(3,277)=$ $1.19, n s, \eta_{p}{ }^{2}=0.013,1-\beta=0.319$. En conclusión, los delincuentes juveniles comparten por igual el distrés psicológico.

\section{Estado clínico (trastornos y sintomas asociados)}

Llevado a cabo un MANOVA 2 (tipo de delito: con violencia vs. sin violencia) X 2 (reincidencia: primarios vs. reincidentes), los resultados no mostraron un efecto significativo en la psicopatología modulado por el factor tipo de delito, $F(9,271)=$ $1.35, n s, \eta_{p}^{2}=0.043,1-\beta=0.507$, por el factor reincidencia, $F(9,271)=1.74, n s, \eta_{p}^{2}=0.055,1-\beta$ $=.786$, ni por la interacción de ambos, $F(9,271)$ $=1.53, n s, \eta_{p}^{2}=0.483,1-\beta=0.72$, esto es, los delincuentes juveniles primarios y reincidentes, $\mathrm{y}$ sentenciados por delitos violentos y no violentos, comparten el mismo estado clínico y los mismos mecanismos internos subyacentes a la delincuencia: ira/hostilidad (escala Hostilidad), ideas persecutorias (escala Ideación Paranoide) y sentimientos 
de inferioridad e inadecuación (escala Sensibilidad Interpersonal).

\section{Manipulación de las respuestas}

Una de las características vinculadas sistemáticamente con la personalidad disocial (Criterio A11 en el DSM-V) y antisocial (Criterio A2 en el DSM-V), es la mentira y, por extensión, la simulación (APA, 2013). Para contrastar este supuesto se procedió con un ANOVA 2 (tipo de delito: con violencia vs. sin violencia) X 2 (reincidencia: primarios vs. reincidentes), obteniéndose un efecto no significativo para el factor tipo de delito en la sinceridad en las respuestas (factor Sinceridad del BAS-3), F(1, 279) $=0.08, n s, \eta_{p}{ }^{2}=0,1-\beta=0.059$, para el factor reincidencia, $F(1,279)=2.6, n s, \eta_{\mathrm{p}}{ }^{2}=0.008,1-\beta=$ 0.322 , y para la interacción de ambos factores, $F(1$, 279) $=0.11, n s, \eta_{\mathrm{p}}^{2}=0,1-\beta=0.062$. Por su parte, la aplicación de los protocolos de evaluación de la simulación no mostró diferencias en la probabilidad de simulación entre condenados por delitos violentos y no violentos, $\chi^{2}(1, N=283)=1.96, n s, \Phi=$ -0.083, pero sí, aunque con un tamaño del efecto pequeño, entre primarios y reincidentes, $\chi^{2}(1, N=$ 283) $=11.1, p<0.001, \Phi=-0.198$, de modo que los reincidentes presentan una mayor probabilidad de simulación (0.165) que los primarios (0.04).

\section{Discusión}

Los anteriores resultados presentan unas limitaciones que han de ser consideradas a la hora de su generalización. Primera, se asume una relación directa entre incompetencia cognitiva y comportamental, y comportamientos delictivos. Si bien generalmente esto es así, también existen individuos con comportamientos delictivos y con una alta competencia, y otros con baja competencia no vinculada a comportamientos delictivos. Segunda, los anteriores resultados no implican necesariamente el establecimiento de una relación causa-efecto. Tercera, los resultados, que se presentan para cada variable por separado, es muy probable que tengan efectos acumulativos en términos de (in)competencia (Andrews \& Bonta, 2010; Fréchette \& LeBlanc, 1987).
Cuarta, las peculiaridades de los instrumentos de medida, así como las propiedades psicométricas, pueden mediar la generalización de los resultados a otros instrumentos. Quinta, la etiqueta judicial de la delincuencia no refleja la realidad de la delincuencia. Sexta, la clasificación de los menores infractores en los niveles de los factores (primarios vs. reincidentes; delitos con violencia vs. sin violencia) se basó en registros oficiales (ground truh) que no son totalmente equiparables a otras medidas como los autoinformes por lo que los resultados han de generalizarse con cautela a otras medidas de dichos niveles (Chereji, Pintea \& David, 2012). Asimismo, el delincuente no se comporta permanentemente como tal, sino que los comportamientos delictivos forman parte de su repertorio conductual a la hora de resolver situaciones sociales problemáticas.

Con estas consideraciones en mente, de los anteriores resultados se pueden extraer las siguientes implicaciones:

a) La gravedad delictiva y, por extensión, del trastorno disocial (referido a los niveles moderado y grave) es totalmente independiente de la competencia cognitiva y comportamental. En consecuencia, la gravedad del acto delictivo no ampara, per se, una intervención judicial más larga motivada en las necesidades del tratamiento, tal y como se argumenta en diversos tratados legales a la hora de justificar la longitud de la condena.

b) La delincuencia crónica o trastorno disocial, entendido como un patrón de comportamiento persistente y repetitivo, descansa, frente a la ocasional, en cierta menor competencia cognitiva y comportamental; mayor inadaptación escolar y familiar y un menor autoncontrol en las relaciones sociales. En consecuencia, la continuidad en la carrera delictiva (reincidencia) se refleja en una mayor inadaptación escolar y social. Por lo tanto, los programas reeducativos que se deben aplicar con delincuentes juveniles reincidentes han de prestar especial atención a la adaptación social y escolar, al tiempo que los programas con los primarios deben 
tener presente que la potenciación de la adaptación escolar y social, actúa como un protector robusto frente a la reincidencia. Asimismo, los delincuentes crónicos han internalizado, en detrimento del acatamiento de las normas y reglas sociales, un patrón de conductas agresivas, impositivas, de terquedad e indisciplina. Dado que dicho patrón se relaciona con la cronicidad delictiva, los programas con los reincidentes han de dirigirse a su neutralización, en tanto que con los primarios a la prevención de su instauración (Chereji et al., 2012). Sin embargo, en una lectura superficial de los resultados, los delincuentes juveniles primarios, frente a los crónicos, no solo no gozarían de ese protector que proporciona el autoconcepto escolar y familiar, sino que los convertiría en más vulnerables. Ahora bien, dado que los crónicos muestran una mayor inadaptación en estas dos mismas áreas (resultado que entra en contradicción con un autoconcepto más positivo) y que un autoconcepto bajo es un rasgo distintivo de la delincuencia, ha de entenderse que, en línea con las características del trastorno disocial, los delincuentes juveniles crónicos están aparentando una imagen de dureza (APA, 2013) o bien que los delincuentes persistentes se refuerzan (se autoevalúan como competentes) fortaleciendo el autoconcepto (Fréchette \& LeBlanc, 1987). Por ello, la intervención con los delincuentes juveniles persistentes ha de dirigirse primeramente a la neutralización de esta disfunción en la percepción que tienen de su autoconcepto, y, posteriormente, a instaurar un autoconcepto realmente positivo que los proteja frente a la reincidencia delictiva (Fréchette \& LeBlanc, 1987). Por su parte, en los primarios procederá la consolidación de un autoconcepto prosocial positivo que lo proteja (Arce et al., 2011). A nivel penal, estas extraordinarias necesidades de los delincuentes juveniles crónicos prestan amparo a una mayor duración de las penas para éstos que para los primarios ante la misma acción delictiva, a fin de que se pueda implementar un tratamiento ajustado a sus mayores carencias y necesidades.

c) La población de delincuentes juveniles, con independencia del tipo de delito cometido y de que sean primarios o crónicos, presenta como característica distintiva niveles uniformes de regulación emocional (inteligencia emocional), población que, acorde a la literatura (Andrews \& Bonta, 2010; Balkin, Miller, Ricard, Garcia \& Lancaster, 2011; Penney \& Moretti, 2010), carece de la habilidad para la evaluación y expresión de las emociones o sentimientos; para la identificación de las emociones y sentimientos experimentados y para el control de las emociones. En consecuencia, todo programa de tratamiento de delincuentes juveniles ha de incluir entre sus objetivos y contenidos la provisión de competencia para la regulación de las emociones.

d) Asimismo, la población de delincuentes juveniles, bien sean primarios o crónicos, o hayan cometido un tipo de delito u otro, comparten capacidad para la resolución de problemas (afrontamiento) que, se debe tener presente, se rige por el uso de estrategias de afrontamiento desadaptativas (Arce et al., 2011; Feelgood, Cortoni \& Thompson, 2005). Por tanto, los programas de tratamiento de delincuentes juveniles han de incluir entre sus objetivos y contenidos dotarlos de competencia en el manejo de estrategias de afrontamiento adaptativas y en el control de las desadaptativas.

e) De igual modo, la población de delincuentes juveniles recurre por un igual a técnicas de neutralización a posteriori del comportamiento delictivo (atribución), y comparten los mismos mecanismos internos subyacentes a la delincuencia que le prestan soporte a priori (rabia/hostilidad, ideas persecutorias, sentimientos de inferioridad e inadecuación, distrés psicológico). Así pues, todo programa de tratamiento de delincuentes juveniles ha de incluir entre sus objetivos y contenidos la 
neutralización de los procesos que soportan $a$ priori y a posteriori el comportamiento delictivo (Novo et al., 2012), y que se relacionan con las recaídas (Isorna, Fernández-Ríos $\&$ Souto, 2010), prestando de este modo apoyo a la prescripción del modelo IDIM de un módulo de intervención dirigido a la neutralización de las fuentes de soporte de la desviación (Fréchette \& LeBlanc, 1987).

f) Los resultados apuntan a que los delincuentes juveniles participan por igual de la mentira y que el tipo de delito cometido no media diferencias en la simulación, pero no así la cronicidad delictiva, de modo que los reincidentes se caracterizan por mayores niveles de simulación. Dado que simulación y mentira son distintivas del trastorno disocial (APA, 2013) y de la población de delincuentes juveniles frente a la población no delictiva (Arce et al., 2011), los programas de intervención han de incluir procedimientos de control de la adherencia y progreso en el tratamiento, ya que puede ser falso o simulado. Esta cautela ha de extremarse entre los crónicos. La simulación y engaño en la adherencia y progreso en el tratamiento bien puede ser la causa por la que fracasan programas cognitivo-conductuales en el manejo de las distorsiones cognitivas (p. ej., atribución externa de la responsabilidad; negación/minimización/justificación de los hechos) (Redondo, Martínez-Catena \& Andrés-Pueyo, 2012). Para el control de esta contingencia contamos con los protocolos de evaluación de la (di)simulación, ajustados al sistema de justicia inquisitorial, que es el propio de la comunidad latinoamericana y de la Europa continental (Arce, Fariña, Carballal \& Novo, 2006, 2009; Arce, Pampillón \& Fariña, 2002; Vilariño, Arce \& Fariña, 2013; Vilariño et al., 2009).

g) Los hallazgos de este estudio respaldan los modelos de cascada/escalada en el desarrollo que asocian niveles de problemas de conducta con niveles de (in)competencia (Hawley, 2003; Masten \& Cicchetti, 2010), y, por extensión, aquellos que relacionan en general incompetencia y comportamientos antisociales y delictivos (e. g., Andrews \& Bonta, 2010; Lösel \& Bender, 2003). Dicho respaldo se concreta en la reincidencia, de modo que entre los delincuentes crónicos se evidencia un efecto de cascada hacia la incompetencia. Sin embargo, los resultados no prestan un apoyo a la generalización de esta correspondencia entre niveles de comportamiento delictivo con los trastornos y síntomas asociados ni a las tipologías de delitos. En resumen, las previsiones de los modelos de cascada/escala en el desarrollo son válidas para la explicación de carencias moderadas por la reincidencia en la aptitud cognitiva y comportamental, pero no así para las tipologías delictivas y clínico-biológicas relacionadas con la delincuencia.

\section{Referencias}

American Psychiatric Association. (2013). Diagnostic and statistical manual of mental disorders (5a. ed.). Washington, DC: Autor.

Andrews, D. A. \& Bonta, J. (2010). The psychology of criminal conduct (5a. ed.). Cincinnati, $\mathrm{OH}$ : Anderson Publishing Co.

Andershed, H., Gustafson, S. B., Kerr, M. \& Stattin, H. (2002). The usefulness of self-reported psychopathy-like traits in the study of antisocial behaviour among non-referred adolescents. European Journal of Personality, 16(5), 383-402.

Arce, R., Fariña, F., Carballal, A. \& Novo, M. (2006). Evaluación del daño moral en accidentes de tráfico: desarrollo y validación de un protocolo para la detección de la simulación. Psicothema, 18(2), 278-283.

Arce, R., Fariña, F., Carballal, A. \& Novo, M. (2009). Creación y validación de un protocolo de evaluación forense de las secuelas psicológicas de la violencia de género. Psicothema, 21(2), 241-247.

Arce, R., Fariña, F. \& Vázquez, M. J. (2011). Grado de competencia social y comportamientos antisociales delictivos y no delictivos en menores. Revista Latinoamericana de Psicología, 43(3), 173-486. 
Arce, R., Pampillón, M. C. \& Fariña, F. (2002). Desarrollo y evaluación de un procedimiento empírico para la detección de la simulación de enajenación mental en el contexto legal. Anuario de Psicología, 33(3), 385-408.

Arce, R., Seijo, D., Fariña, F. \& Mohamed-Mohand, L. (2010). Comportamiento antisocial en menores: riesgo social y trayectoria natural de desarrollo. Revista Mexicana de Psicología, 27(2), 127-142.

Balkin, R. S., Miller, J., Ricard, R. J., Garcia, R. \& Lancaster, C. (2011). Assessing factors in adolescent adjustment as precursors to recidivism in courtreferred youth. Measurement and Evaluation in Counseling and Development, 44(1), 52-59.

Binswanger, I. A., Merrill, J. O., Krueger, P. M., White, M. C., Booth, R. E. \& Elmore, J. G. (2010). Gender differences in chronic medical, psychiatric, and substance-dependence disorders among jail inmates. American Journal of Public Health, 100(3), 476-482.

Chereji, S. V., Pintea, S. \& David, D. (2012). The relationship of anger and cognitive distortions with violence in violent offenders' population: A metaanalytic review. The European Journal of Psychology Applied to Legal Context, 4(1), 59-77.

Cohen, J. (1998). Statistical power analysis (2a. ed.). Hillsdale, NJ: LEA.

Contreras, L., Molina, V. \& Cano, M. C. (2011). In search of psychological variables linked to the recidivism in young offenders. The European Journal of Psychology Applied to Legal Context, 3(1), 77-88.

Declercq, F., Markey, S., Vandist, K. \& Verhaeghe, P. (2009). The youth psychopathic trait inventory: Factor structure and antisocial behaviour in nonreferred 12-17-year-olds. Journal of Forensic Psychiatry $\mathcal{E}$ Psychology, 20(4), 577-594.

Elrod, P. \& Ryder, R. S. (2011). Juvenile justice: A social, historical, and legal perspective (2a. ed.). Sudburry, MA: Jones and Bartlett Publishers.

Feelgood, S., Cortoni, F. \& Thompson, A. (2005). Sexual coping, general coping, and cognitive distortions in incarcerated rapist and child molesters. Journal of Sexual Aggression, 11(2), 157-170.

Fernández-Berrocal, P., Extremera, N. \& Ramos, N. (2004). Validity and reliability of the Spanish modified version of the Trait Meta-Mood Scale. Psychological Reports, 94(3), 751-755.

Fréchette, M. \& LeBlanc, M. (1987). Délinquances et délinquants. Chicoutimi, Quebec, Canadá: Gaëtan Morin.

Frick, P. J., Stickle, T. R., Dandreaux, D. M., Farrel J. M. \& Kimonis, E. R. (2005). Callous-unemotional traits in predicting the severity and stability of conduct problems and delinquency. Journal of $A b$ normal Child Psychology, 33(4), 471-487.

Frydenberg, E. \& Lewis, R. (2000). Escalas de afrontamiento para adolescentes (ACS). Madrid: TEA Ediciones.

Gázquez, J. J., Pérez-Fuentes, M. C., Carrión, J. J. \& Santuiste, V. (2010). Estudio y análisis de conductas violentas en Educación Secundaria en España. Universitas Psychologica, 9(2), 371-380.

Halty, L., Martínez, A., Requena, C., Santos, J. M. \& Ortiz, T. (2011). Psicopatía en niños y adolescentes: modelos, teorías y últimas investigaciones. Revista Neurología, 52(Supl.), S19-S27.

Hawley, P. H. (2003). Prosocial and coercitive configurations of recource control in early adolescence: A case for the well-adapted Machiavellian. MerrillPalmer Quarterly, 49(3), 279-309.

Hernández, P. (2002). Test autoevaluativo multifactorial de adaptación infantil (TAMAI) (3a. ed.). Madrid: TEA Ediciones.

Howard, R. (2009). The neurobiology of affective dyscontrol: Implications for understanding "dangerousness and severe personality disorder”. En M. McMurran \& R. Howard (Eds.), Personality, personality disorder and violence (pp. 157-174). Chichester, UK: John Wiley and Sons.

Indiana Department of Correction. (2010). Juvenile recidivism rates, 2010 (Informe). Indianapolis, IN: Autor. Recuperado el 14 de julio de 2012, de http:// www.in.gov/idoc/files/2010JuvRecidivismRpt.pdf

Isorna, M., Fernández-Ríos, L. \& Souto, A. (2010). Treatment of drug addiction and psychopathology: A field study. The European Journal of Psychology Applied to Legal Context, 2(1), 3-18.

Ley Orgánica 5/2000, de 12 de enero, reguladora de la responsabilidad penal de los menores. BOE, 11, 1422-1441. 
Ley Orgánica 8/2006, de 4 de diciembre, por la que se modifica la Ley Orgánica 5/2000, de 12 de enero, reguladora de la responsabilidad penal de los menores. BOE, 290, 42700-42712.

Lösel, F. \& Bender, D. (2003). Protective factors and resilience. En D. P. Farrington \& J. W. Coid (Eds.), Early prevention of antisocial behaviour (pp. 130. 204). Cambridge, UK: Cambridge University Press.

Lösel, F. \& Farrington, D. P. (2012). Direct protective and buffering protective factors in the development of youth violence. American Journal of Preventive Medicine, 43(Suppl. 1), 63-68.

Lynam, D. R., Miller, D. J., Vachon, D., Loeber, R. \& Stouthamer-Loeber, M. ( 2009). Psychopathy in adolescence predicts official reports of offending in adulthood. Youth Violence and Juvenile Justice, 7(3), 189-207.

Marsee, M. A., Silverthorn P. \& Frick, P. J. (2005). The association of psychopathic traits with aggression and delinquency in non-referred boys and girls. Behavioral Sciences and the Law, 23(6), 803-817.

Maruna, S. \& Mann, R. E. (2006). A fundamental attribution error? Rethinking cognitive distortions. Legal and Criminological Psychology, 11(2), 155-177.

Masten, A. S. \& Cicchetti, D. (2010). Developmental cascades. Development and Psychopathology, 22(3), 491-495.

McMahon, R. J., Wells, K. C. \& Kotler, J. S. (2006). Conduct problems. En E. J. Mash \& R. A. Barkley (Eds.), Treatment of childhood disorders (3a. ed., pp. 137-268). New York: The Guilford Press.

McMahon, R. J., Witkiewitz, K. \& Kotler, J. S. (2010). Predictive validity of callous-unemotional traits measured in early adolescence with respect to multiple antisocial outcomes. Journal of Abnormal Psychology, 119(4), 752-763.

McGuire, J. (2008). A review of effective interventions for reducing aggression and violence. Philosophical Transactions of the Royal Society: Biological Sciences, 363(1503), 2577-2597.

Moffitt, T. E. (1993). Adolescence-limited and lifecourse persistent antisocial behavior: A developmental taxonomy. Psychological Review, 100(4), 674-701.
Muñoz, L. C., Kerr, M. \& Besic, N. (2008). The peer relationships of youths with psychopathic personality traits. Criminal Justice and Behavior, 35(2), 212-227.

Musitu, G., García, F. \& Gutiérrez, M. (1997). Autoconcepto forma A (3a. ed.). Madrid: TEA Ediciones.

Novo, M., Fariña, F., Seijo, D. \& Arce, R. (2012). Assessment of a community rehabilitation programme in convicted male intimate-partner violent offenders. International Journal of Clinical and Health Psychology, 12(2), 219-234.

Olson, C. L. (1976). On choosing a test statistic in MANOVA. Psychological Bulletin, 83(4), 579-586.

Penney, S. R. \& Moretti, M. M. (2010). The roles of affect dysregulation and deficient affect in youth violence. Criminal Justice and Behavior, 37(6), 709-731.

Redondo, S., Martínez-Catena, A. \& Andrés-Pueyo, A. (2012). Therapeutic effects of a cognitive-behavioural treatment with juvenile offenders. The European Journal of Psychology Applied to Legal Context, 4(2), 159-178.

Redondo, S., Sánchez-Meca, J. \& Garrido, V. (2002). Los programas psicológicos con delincuentes y su efectividad: la situación europea. Psicothema, 14(Supl. 1), 164-173.

Rhee, S. Y. \& Waldman, I. (2009). Genetic analysis of conduct disorder and antisocial behavior. En Y. K. Kim (Ed.), Handbook of behavior genetics (pp. 455-471). New York: Springer.

Rodríguez, F. J., Bringas, C., Rodríguez, L., López-Cepero, J., Pérez, B. \& Estrada, C. (2011). Drug abuse and criminal family records in the criminal history of prisoners. The European Journal of Psychology Applied to Legal Context, 3(2), 89-105.

Rotter, J. B. (1966). Generalized expectancies of internal versus external control of reinforcement. Psychological Monographs: General and Applied, 80, 1-28.

Serin, R. C. \& Amos, N. L. (1995). The role of psychopathy in the assessment of dangerousness. International Journal of Law and Psychiatry, 18(2), 231-238.

Silva, F. \& Martorel, M. C. (1989). BAS-3: Batería de socialización (autoevaluación) (2a. ed.). Madrid: TEA Ediciones.

Vilariño, M., Arce, F. \& Fariña, F. (2013). Forensicclinical interview: Reliability and validity for the evaluation of psychological injury. The European 


\section{¿ESTÁ MEDIADA LA GRAVEDAD DELICTIVA Y CRONICIDAD DE LOS DELINCUENTES JUVENILES POR LA COMPETENCIA COGNITIVO-COMPORTAMENTAL?}

Journal of Psychology Applied to Legal Context, 5(1), 1-21.

Vilariño, M., Fariña, F. \& Arce, R. (2009). Discriminating real victims from feigners of psychological injury in gender violence: Validating a protocol for forensic settings. The European Journal of Psychology Applied to Legal Context, 1(2), 221-243. 
